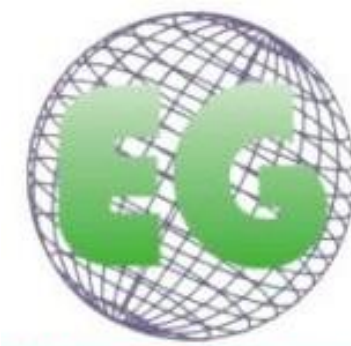

ISSN 1695-6141 $N^{\circ} 56$

\title{
Uso de catéteres venosos de línea media en pacientes hospitalizados
}

The use of venous catheters of average line in hospitalized patient

\author{
Nuria Fortes Escalona ${ }^{1}$ \\ Juana María Fernández Domínguez ${ }^{1}$ \\ Concepción Cruzado Álvarez ${ }^{1}$ \\ Susana García Matez ${ }^{1}$ \\ ${ }^{1}$ Enfermera. Hospital Virgen de la Victoria, Málaga. España. nuriafortes@msn.com
}

\section{http://dx.doi.org/10.6018/eglobal.18.4.334891}

Recibido: 19/06/2018

Aceptado: 3/10/2018

\section{RESUMEN:}

En el contexto actual en el que han surgido numerosas alternativas en terapia intravenosa, se hacen necesarios estudios sobre los diferentes dispositivos disponibles para determinar cuál es el sistema más idóneo en cada caso. Las líneas medias surgen como alternativa a la utilización de catéteres venosos periféricos y catéteres centrales de inserción periférica. El objetivo de este estudio es evaluar el uso de los catéteres de línea media en pacientes hospitalizados.

Método: Estudio retrospectivo de 1016 pacientes. Se analizaron variables relacionadas con el tiempo medio de utilización del catéter, la tasa de incidencias para cada una de las complicaciones, el número de catéteres retirados y sus causas.

Resultados: El $40 \%$ de los catéteres se canalizó por mal acceso venoso del paciente (406), el $42 \%$ por tratamiento intravenoso largo (427) y el $18 \%$ por tratamiento irritante (183). Respecto a la efectividad de los catéteres, el tiempo medio de utilización fue de 12.1 días (DE 9.4). Finalizaron el tratamiento 854 pacientes, $704(69.3 \%)$ por fin de tratamiento y $150(14.8 \%)$ por fallecimiento. Presentaron complicaciones el $7.4 \%$ de los pacientes (75). Se retiraron por mal funcionamiento del catéter el $4.4 \%$ (45), el $1 \%$ por complicaciones del paciente (10). El $2 \%$ de los catéteres se retiró por fiebre (20).

Conclusiones: El catéter de línea media es un dispositivo de acceso venoso con una baja tasa de complicaciones, presenta un tiempo medio de permanencia en torno a los 12 días. Supone una alternativa a los catéteres periféricos cortos en pacientes con mal acceso venoso y ofrece una alternativa a las rotaciones frecuentes en pacientes con tratamientos antibióticos superiores a 7 días.

Palabras clave: dispositivos de acceso venoso; catéter de línea media; midline.

\section{ABSTRACT:}

In the current context in which numerous alternatives have emerged in intravenous therapy, studies are needed on the different devices available to determine which is the most suitable system in each case. The midlines arise as an alternative to the use of peripheral venous catheters and central peripheral insertion catheters. The main objective of this study is to evaluate the use of midline catheters in hospitalized patients. 
Method: Retrospective study of 1016 patients. We analyzed variables related to the mean time of catheter use, the incidence rate for each of the complications, the number of catheters removed and their causes.

Results: $46.5 \%$ of the catheters were inserted in men (472). The average age was 65 years (SD 16.8), being the youngest of 14 and the oldest of 101 years. $40 \%$ of the catheters were channeled due to poor venous access of the patient (406), 42\% for long intravenous treatment (427) and $18 \%$ for irritant treatment (183). Regarding the effectiveness of the catheters, the average time of use was 12.1 days (SD 9.4). The treatment was terminated by 854 patients, 704 (69.3\%) by the end of treatment and 150 $(14.8 \%)$ by death. $7.4 \%$ of patients presented complications (75). $4.4 \%$ (45) were removed due to malfunction of the catheter, $1 \%$ due to patient complications (10). $2 \%$ of the catheters were removed due to fever (20).

Conclusions: The middle line catheter is a venous access device with a low complication rate, it has a average residence time of around 12 days. It is an alternative to short peripheral catheters in patients with poor vein access and offers an alternative to frequent rotations in patients with antibiotic treatments longer than 7 days.

Key words: venous access devices; midline catheter; midline.

\section{INTRODUCCIÓN}

La terapia endovenosa es un tratamiento usual en pacientes hospitalizados. Las perfusiones de fármacos pueden producir flebitis y extravasaciones, por lo que frecuentemente los pacientes son portadores durante el ingreso de varias vías venosas periféricas ${ }^{(1)}$.

Insertar un catéter venoso es un proceso invasivo que además de dolor e incomodidad, genera complicaciones como la flebitis ${ }^{(2,3)}$. Las características de las perfusiones constituyen el principal factor del fallo de las vías periféricas, el personal de enfermería debe adecuar el tipo de catéter venoso a las particularidades del tratamiento prescrito. Dependiendo de la agresividad y duración del tratamiento se utilizarán catéteres venosos periféricos o catéteres venosos centrales. La recomendación general es que se utilicen catéteres venosos centrales para infusiones con $\mathrm{pH}$ inferior a 5 o superior a 9 . Si bien no hay estudios de resultados que sugieran que el $\mathrm{pH}$ por si mismo cause flebitis ${ }^{(4)}$. Existen numerosos estudios que demuestran que son muchos los factores que contribuyen a la tromboflebitis relacionada con la infusión (ubicación anatómica, sexo, experiencia del insertador, etc.) ${ }^{(5)}$.

En los últimos años ha resurgido el uso de los denominados catéteres de línea media (CM), se trata de catéteres venosos de entre $8-25 \mathrm{~cm}$ de longitud. Estos catéteres ofrecen a los pacientes y profesionales la posibilidad de ampliar la duración de la terapia intravenosa, ya que están compuestos de materiales más seguros y biocompatibles como el poliuretano. Son insertados mediante acceso periférico a través de la vena braquial o basílica, hasta localizar la punta del catéter en el sistema venoso profundo (vena axilar o subclavia). Permiten la infusión de tratamientos endovenosos con indicación de administrar a través de vías venosas periféricas cortas, con la ventaja añadida de que pueden durar hasta seis semanas. Según los Centros para el Control y la Prevención de Enfermedades (CDC) "los catéteres de línea media se asocian con tasas más bajas de flebitis que los catéteres periféricos cortos y con tasas de infección más bajas que los centrales" ${ }^{(6-10)}$.

La técnica de inserción mediante micropunción guiada por ultrasonido ha supuesto también, un avance importante para atajar los problemas relacionados con los accesos venosos en el ámbito hospitalario ${ }^{(11)}$. Esta técnica, aumenta el porcentaje de éxito y por tanto disminuye el número de venopunciones. 
A diferencia de los catéteres de inserción central en los que para su colocación y retirada se requiere la intervención de médicos, los catéteres de línea media pueden ser insertados y retirados por profesionales de enfermería.

Por todo ello, en los últimos años el uso de los CM se ha intensificado, tanto para la terapia intravenosa en infusión continua como intermitente. El objetivo principal de este estudio fue evaluar el uso de los CM en pacientes hospitalizados.

\section{MÉTODO}

Se realizó un estudio retrospectivo de todos los pacientes a los que se había canalizado un CM en la Unidad de Terapia Intravenosa (UTIV). Entre el 16 de julio de 2015 y el 18 de abril de 2017 se canalizaron 1140 catéteres, se desestimaron aquellos de los que no se disponían de datos relativos a la retirada; quedando la muestra en 1016 pacientes.

Los datos necesarios para este estudio se obtuvieron de la base de datos elaborada por el personal de enfermería de la UTIV. En ella se registraron las características sociodemográficas de los pacientes (sexo, fecha de nacimiento, edad y especialidad), el tipo de tratamiento endovenoso infundido, los datos relativos a la inserción (fecha de inserción, lugar de punción), las complicaciones o incidencias surgidas y el motivo y fecha de retirada. Esta investigación se clasifica en el grupo de sin riesgo según la resolución 008430 de 1993 del Ministerio de Salud.

En cumplimiento con la Ley de Protección de Datos y Documentación Clínica, en el registro no se recogieron datos de carácter personal que permitieran identificar a las personas participantes.

Para evaluar el uso de los CM, se analizaron las indicaciones de inserción de estos catéteres en pacientes hospitalizados, el tiempo medio de utilización, las complicaciones inherentes a su uso y las causas de retirada en relación con el motivo de inserción.

Se consideraron complicaciones relacionadas con el catéter, la obstrucción total o parcial y la rotura. Referidas al paciente, la infección en el punto de punción, fiebre, bacteriemia, flebitis y trombosis. Finalmente se consideraron como otras causas de retirada la extracción accidental por parte del paciente o del personal sanitario.

Los pacientes se dividieron en tres grupos en función de la indicación de canalización de un CM:

- Mal acceso venoso, incluye aquellos pacientes con ausencia de venas superficiales visibles o palpables y/o con fallo de dos o más intentos.

- Pacientes con prescripción de tratamiento antibiótico superior a una semana.

- Pacientes con indicación de tratamiento intravenoso considerados irritantes por su osmolaridad $\mathrm{y} / \mathrm{o} \mathrm{pH}$. 


\section{Análisis estadístico}

Se realizó un análisis descriptivo tanto de las variables sociodemográficas básicas de los pacientes como de las características de los catéteres insertados. Asimismo, se llevó a cabo una primera aproximación de carácter descriptivo del tiempo de utilización de los catéteres implantados y del motivo de retirada. En función de la naturaleza de las variables se han utilizado prueba de chi cuadrado, t de Student o ANOVA.

\section{RESULTADOS}

Los resultados del análisis descriptivo quedan recogidos en la tabla 1. Se analizaron 1016 catéteres, el $46.5 \%$ se insertaron en hombres (472). La edad media fue de 65 años (DE 16.8), siendo el más joven de 14 y el mayor de 101. El $40 \%$ de los catéteres se canalizaron por mal acceso venoso (406), el $42 \%$ por tratamiento intravenoso largo (427) y el 18\% por tratamiento irritante (183). El brazo de elección fue el derecho en el $80.6 \%$ de los pacientes (819) y en el $94.8 \%$ de los casos se canalizó la vena basílica (963).

Por especialidades, las unidades que solicitan un CM en mayor porcentaje son: Medicina Interna (23.7\%), Cirugía (18.3\%), Digestivo (12.6\%), Enfermedades Infecciosas (8.7\%), Cirugía Traumatológica (8.3\%), Neurología (6.8\%), Neumología $(5.2 \%)$.

Los tratamientos irritantes administrados en mayor porcentaje fueron: Isoplasmal $30.6 \%$ (56), Cloruro potásico 19.1\% (35), Vancomicina 12\% (22), Cloxacilina 9.3\% (17).

El tiempo medio de utilización de los CM fue de 12.1 días (DE 9.4). En los pacientes con mal acceso la media fue de 12.3 días (95\% IC 11.2-13.3), en el grupo de tratamiento largo de 11.5 días (95\%IC 10.7-12.3) y en los de tratamiento irritante de 13 días (95\% IC 13-11.5). Las diferencias entre grupos no fueron significativas $(\mathrm{p}=0.15)$.

Finalizaron el tratamiento 854 pacientes, 704 (69.3\%) por fin de tratamiento y 150 (14.8\%) por fallecimiento. Por grupos, finalizan el tratamiento el $68.5 \%$ de los pacientes con mal acceso venoso (278), el $71 \%$ de los que tenían tratamiento largo (303) y el $65.6 \%$ con tratamiento irritante (120).

Tabla 1: Características de la muestra

\begin{tabular}{|l|l|l|}
\hline $\mathrm{N}=1016$ & & \\
\hline Sexo (hombre) & 472 & $46.5 \%$ \\
\hline Edad & 65 años & 16.8 \\
\hline Brazo (derecho) & 819 & $80.6 \%$ \\
\hline Vena (basílica) & 963 & $94.8 \%$ \\
\hline Tiempo de Implantación & 12.1 & 9.4 \\
\hline Indicación del catéter & & \\
\hline Mal acceso venoso & 406 & $40 \%$ \\
\hline Tratamiento de larga duración (> 7 días) & 427 & $42 \%$ \\
\hline Tratamiento irritante & 183 & $18 \%$ \\
\hline UGC & & \\
\hline Medicina Interna & 241 & $23.7 \%$ \\
\hline
\end{tabular}




\begin{tabular}{|l|l|l|}
\hline Cirugía Digestiva & 186 & $18.3 \%$ \\
\hline Digestivo & 128 & $12.6 \%$ \\
\hline Enfermedades Infecciosas & 88 & $8.7 \%$ \\
\hline Traumatología & 84 & $8.3 \%$ \\
\hline Neurología & 69 & $6.8 \%$ \\
\hline Neumología & 52 & $5.1 \%$ \\
\hline Oncología & 45 & $4.4 \%$ \\
\hline Angiología & 44 & $4.3 \%$ \\
\hline Otras Unidades & 79 & $7.8 \%$ \\
\hline Causas de retirada & & \\
\hline Fin de tratamiento & 704 & $69.3 \%$ \\
\hline Exitus & 150 & $14.8 \%$ \\
\hline Mal funcionamiento del catéter & 45 & $4.4 \%$ \\
\hline Cambio por central & 42 & $4.1 \%$ \\
\hline Fiebre & 20 & $2.0 \%$ \\
\hline Complicaciones del paciente & 10 & $1 \%$ \\
\hline Renovación & 3 & $0.3 \%$ \\
\hline Otros & 42 & $4.1 \%$ \\
\hline Tratamientos irritantes & & \\
\hline Isoplasmal & 56 & $30.6 \%$ \\
\hline Cloruro Potásico & 35 & $19.1 \%$ \\
\hline Vancomicina & 22 & $12 \%$ \\
\hline Cloxacilina & 17 & $9.3 \%$ \\
\hline Gentamicina & 8 & $4.4 \%$ \\
\hline Alprostadil & 7 & $3.8 \%$ \\
\hline Clindamicina & 5 & $2.7 \%$ \\
\hline Daptomicina & 5 & $2.7 \%$ \\
\hline Bicarbonato & 3 & $1.6 \%$ \\
\hline Otros & 25 & $13.8 \%$ \\
\hline & 0 & \\
\hline
\end{tabular}

Las cantidades representan frecuencias (\%) o bien media (DE)

Dentro del grupo de pacientes de tratamiento largo se identificó a un grupo de 143 pacientes con cirugía programada de colon. A los que se les canalizaba un CM el primer día de hospitalización previo a la cirugía. El $90.2 \%$ (129) finalizaron el tratamiento sin complicaciones, sólo el $3.5 \%$ (6) precisó canalizar un catéter venoso central.

Presentaron complicaciones el $7.4 \%$ de los pacientes (75). El $4.4 \%$ (45) relacionadas con mal funcionamiento del catéter, el $2 \%$ (20) relacionadas con fiebre y el $1 \%$ (10) fueron complicaciones del paciente.

Cuando analizamos la relación entre indicación del catéter y complicaciones, encontramos que los pacientes a los que se le canalizaba por mal acceso venoso tenían un $4.7 \%$ de complicaciones (19), mientras que aquellos en los que la indicación era por tratamiento largo presentaba un 9.1\% de complicaciones (39) y los de tratamiento irritante un $9.3 \%$ (17). Siendo la diferencia estadísticamente significativa $p=0.027$.

Los pacientes que precisaron cambiar el $\mathrm{CM}$ por un catéter venoso central representaban el $2.2 \%$ en el grupo de mal acceso venoso (9), el $4.2 \%$ en el de tratamiento largo (18) y el $7.1 \%$ en el de tratamiento irritante (13). La diferencia fue estadísticamente significativa, $\mathrm{p}=0.017$.

En la figura 1 pueden verse las causas de retirada. Se retiraron por mal funcionamiento del catéter el $4.4 \%$ (45), el $1 \%$ por complicaciones en el paciente 
(10), el $4.1 \%$ (42) por necesidad de un acceso venoso central. El $2 \%$ de los catéteres se retiró por fiebre (20). En todos los casos se realizó cultivo de la punta, sólo 7 fueron positivos. A 3 pacientes se les canalizó un nuevo CM para finalizar el tratamiento.

Figura 1: Causas de retirada

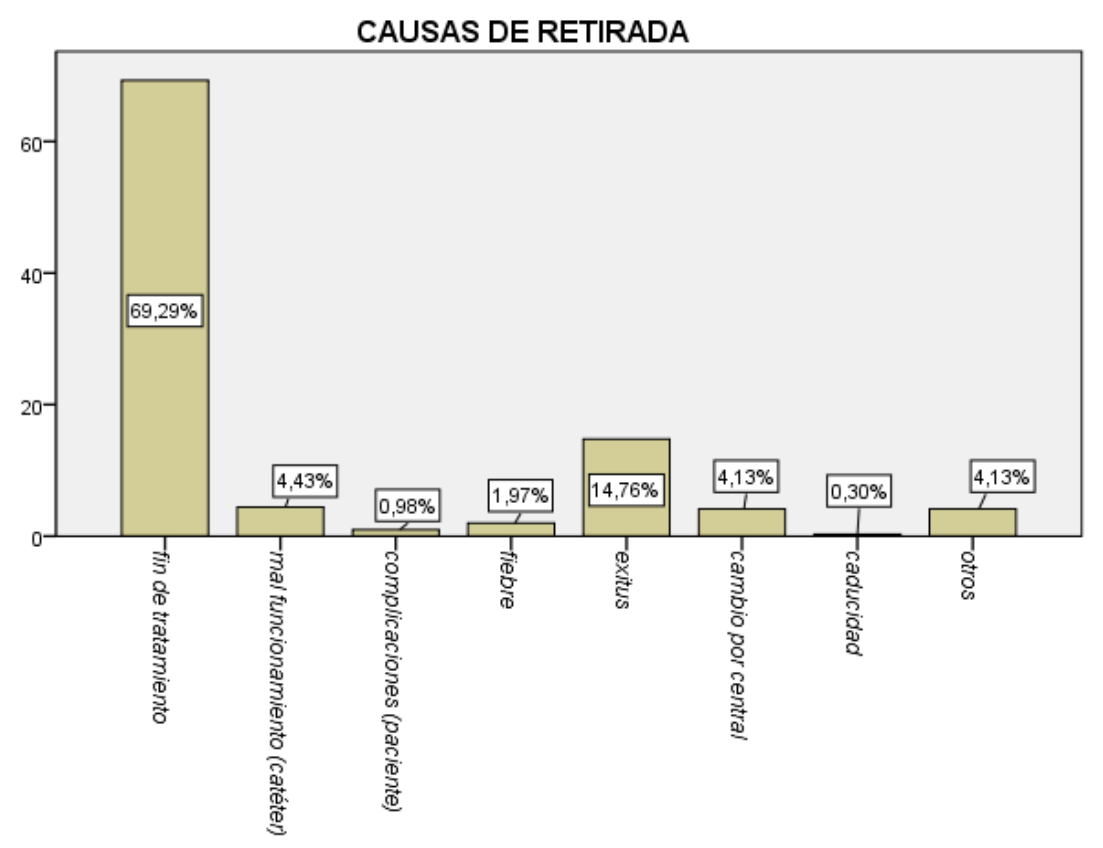

\section{DISCUSIÓN}

Los CM se utilizan actualmente en el sistema sanitario aunque con variabilidad según centros e indicaciones. En nuestro hospital su consumo como dispositivo de acceso venoso se ha incrementado exponencialmente desde la puesta en funcionamiento de la UTIV, tanto el personal sanitario como los pacientes han mostrado un alto grado de satisfacción con los mismos, ya que preserva el capital venoso y disminuye el sufrimiento del paciente evitando múltiples venopunciones.

El catéter periférico corto es el de elección en caso de urgencia inmediata, pero no está recomendado para la perfusión de productos de alta osmolaridad o elevada capacidad irritativa. En estos casos los catéteres de línea media se muestran como una alternativa eficiente para pacientes con tratamientos antibióticos endovenosos superiores a una semana (7) (10). En nuestro estudio el tiempo medio de utilización de los CM fue de 12.1 días y sólo el $7.4 \%$ de los pacientes presentaron complicaciones. Este porcentaje puede verse reducido con programas formativos ya que el $4.4 \%$ de las mismas se relacionan con el manejo del catéter.

Los datos relacionados con los pacientes de cirugía de colon, confirman que la canalización de un CM de forma temprana en pacientes que requieren hospitalización prolongada, es una opción costoefectiva ${ }^{(11)}$.

La literatura reciente indica que el alcance de los CM puede estar en expansión. Un estudio realizado por Caparas et al. mostró la seguridad de la administración de vancomicina a través de un CM versus un PICC ${ }^{(12)}$. En nuestro estudio no encontramos diferencia en cuanto a las complicaciones entre pacientes a los que se 
les administró tratamiento antibiótico con bajo riesgo de flebitis y aquellos en los que el tratamiento era irritante. En todos los casos se siguieron las recomendaciones del servicio de Farmacia respecto a dilución del fármaco y velocidad de perfusión. Consideramos que se deben tener en cuenta como factores asociados a la aparición de complicaciones, además de las características intrínsecas del fármaco (osmolaridad), la velocidad de administración y la duración del tratamiento. Otro factor importante es el tiempo de perfusión de la solución administrada ya que en soluciones de osmolaridad elevada el riesgo de aparición de flebitis para perfusiones prolongadas es mayor que para soluciones administradas en bolus ${ }^{(13)}$.

Sólo el 7\% de los pacientes con tratamiento irritante precisaron canalizar una vía central. Se trataba principalmente de pacientes en tratamiento con isoplasmal que por su evolución clínica precisaron nutrición parenteral. Por tanto los CM se muestran como una alternativa en pacientes que precisan tratamientos irritantes y en los que no se justifica la canalización de una vía venosa central como primera opción. La tasa global de complicaciones durante la canalización de un catéter venoso central se estima en torno al 15\%, por lo que se debe considerar su canalización sólo en casos absolutamente necesarios ${ }^{(14-16)}$.

El coste de los CM, en torno a los 30 euros, hace que estos dispositivos sean cotoefectivos respecto a los catéteres venosos centrales de inserción periférica ${ }^{17}$. Los denominados PICC tienen un precio elevado por lo que se debe sopesar su utilización en terapias de pocos días de duración.

\section{Limitaciones}

Se trata de un estudio retrospectivo de un solo centro, no un ensayo clínico aleatorizado. Se estudiaron las complicaciones ocurridas durante la estancia hospitalaria, por lo que podrían faltar datos sobre complicaciones que ocurrieron tras el alta hospitalaria.

Finalmente, se hacen necesarios estudios sobre el impacto potencial en función del tipo de catéter y del fabricante.

\section{¿Qué se conoce?}

Los MC se remontan a la década de 1950, las reacciones de hipersensibilidad al material del catéter en ciertos diseños condujeron a una disminución en su fabricación y uso. Después de un rediseño de materiales y métodos de inserción, han vuelto a ganar popularidad como un dispositivo alternativo de acceso venoso periférico.

El uso de $\mathrm{MC}$ es compatible con pacientes que requieren terapia intravenosa a mediano y largo plazo. Permite una mayor hemodilución de los medicamentos administrados, reduciendo la incidencia de flebitis química, infiltración y malestar del paciente con la administración del fármaco. El tiempo potencial de permanencia de hasta 28 días, sugiere su colocación temprana en el curso de la hospitalización en pacientes que se espera necesiten infusiones intravenosas prolongadas o acceso venoso difícil. Mientras que algunos estudios sugieren que las soluciones de medicación irritante no pueden administrarse a través de $\mathrm{CM}$, estudios recientes indican lo contrario. 


\section{¿Qué aporta?}

El CM es un dispositivo de acceso venoso con una baja tasa de complicaciones, presenta un tiempo medio de permanencia en torno a los 12 días. Se muestran como una opción para pacientes que requieren tratamiento endovenosos durante la hospitalización. Supone una alternativa a los catéteres periféricos cortos en pacientes con mal acceso venoso y disminuye el riesgo además de proteger el capital venoso de pacientes con tratamientos antibióticos superiores a 7 días. Especialmente cuando se usan en una población de pacientes debidamente seleccionada.

Finalmente los $\mathrm{CM}$ suponen también una alternativa segura para los pacientes con indicación de tratamiento irritante en los que no se justifica la canalización de un catéter venoso central.

\section{REFERENCIAS}

1. Cheung E, Baerlocher MO, Asch M, Myers A. Venous access: a practical review for. Can Fam Physician. 2009;55:494-496.

2. Carrero Caballero MC, Montealegre Sanz M, Cubero Pérez MA. Catéter venoso medial o midline. Revista Rol de Enfermería. 2014;37(1):36-41.

3. Arias-Fernández L, Suérez-Mier B, Martínez-Ortega $M$ del $C$, Lana A. Incidencia y factores de riesgo de flebitis asociadas a catéteres venosos periféricos. Enferm Clin. 2017;27:79-86.

4. Griffiths V. Midline catheters: indications, complications and maintenance Nurs Stand. 2007;22:48-58.

5. McGoldrick METRO Prevención y control de infecciones. En: Alejandro METRO Corrigan UN Gorski L Infusión de enfermería: un enfoque basado en la evidencia. $3^{\underline{a}}$ ed. St. Louis, MO Saunders / Elsevier 2010 204-228.

6. Roszell S, Jones C. Problemas de administración intravenosa. JIN.2010; 33:112-118.

7. O'Grady NP, Alexander M, Burns LA, Dellinger EP, Garland J, Heard SO, et al. Guidelines for the prevention of intravascular catheter-related infections. Am J Infect Control. 2011 May;39(4 Suppl 1):S1-34.

8. Adams DZ, Little A, Vinsant C, Khandelwal S. The Midline Catheter: A Clinical Review. J Emerg Med. 2016 Sep;51(3):252-8.

9. Intravenous Nurses Society. Position paper: midline and midclavicular catheters. J Intraven Nurs. 1997;20:175-178.

10. The Use of Midline Catheters in the Adult Acute Care Setting - Clinical Implications and Recommendations for Practice. Journal of the Association for Vascular Access. 2011;16(1):35-41.

11. G. Scoppettuolo, M. Pittiruti, L. Dolcetti, et al. Ultrasound-guided "short" midline catheters for difficult venous access in the emergency department: a retrospective analysis. Int J Emerg Med. 2016;9:3.

12. J.V. Caparas JV, Hu JP. Safe administration of vancomycin through a novel midline catheter: a randomized, prospective clinical trial. $\mathrm{J}$ Vasc Access.2014;15:251-256.

13.. LaRue GD, Peterson M. The impact of dilution on intravenous therapy. J Infus Nurs. 2011 Apr;34(2):117-23.

14. McGee DC, Gould MK. Preventing complications of central venous catheterization. N Engl J Med.2003;348:1123. 
15. Chopra V, O'Horo JC, Rogers MA, Maki DG, Safdar N. The risk of bloodstream infection associated with peripherally inserted central catheters compared with central venous catheters in adults: a systematic review and meta-analysis. Infect Control Hosp Epidemiol. 2013;34:908-918.

16 Dawson RB, Moureau NL. Midlines: an essential tool in CLABSI reduction Infect Control Today. 2013;17:42-45.

17. Pathak R, Patel A, Enuh H, Adekunle O, Shrisgantharajah V, Diaz K. The Incidence of Central Line-Associated Bacteremia After the Introduction of Midline Catheters in a Ventilator Unit Population. Infect Dis Clin Pract (Baltim Md). 2015 May;23(3):131-4. 